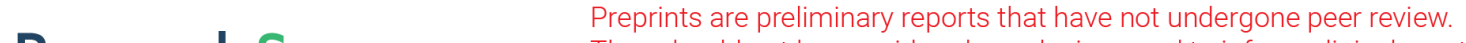 They should not be considered conclusive, used to inform clinical practice, or referenced by the media as validated information. \\ Pulmonary function of patients with 2019 novel coronavirus induced pneumonia: A Retrospective Cohort Study
}

\section{Dongqing LV}

Taizhou Hospital of Zhejiang Province

\section{Xi Chen}

Taizhou Hospital of Zhejiang Province

\section{Linghong Mao}

Taizhou Hospital of Zhejiang Province

Jiao Sun

Taizhou Hospital of Zhejiang Province

\section{Guixian Wu}

Taizhou Hospital of Zhejiang Province

Zhi Lin

Taizhou Hospital of Zhejiang Province

\section{Ronghai Lin}

Taizhou Hospital of Zhejiang Province

Jiansong Yu

Taizhou Hospital of Zhejiang Province

Xiaomai Wu

Taizhou Hospital of Zhejiang Province

Yongpo Jiang ( $\square$ jyongpo8@163.com )

Taizhou Hospital of Zhejiang Province https://orcid.org/0000-0002-5681-3663

\section{Research}

Keywords: COVID-19 infected pneumonia; Pulmonary fibrosis; Pulmonary function; Restrictive ventilation disorder

Posted Date: April 27th, 2020

DOI: https://doi.org/10.21203/rs.3.rs-24303/v1

License: (c) (1) This work is licensed under a Creative Commons Attribution 4.0 International License. Read Full License 
Version of Record: A version of this preprint was published at Annals of Palliative Medicine on September 1st, 2020. See the published version at https://doi.org/10.21037/apm-20-1688. 


\section{Abstract}

Background: Pulmonary fibrosis is a common complication in patients with viral pneumonia, which causes restricted ventilation disorders and affects the prognosis of patients. However, the pulmonary function of patients with 2019 novel coronavirus (COVID-19)-induced pneumonia has not yet been reported.

Methods: A retrospective analysis of 137 patients with COVID-19-induced pneumonia who were discharged from the Enze Hospital, Taizhou Enze Medical Center (Group), from January 31, 2020, to March 11, 2020. Follow-up occurred two weeks after hospital discharge, whereupon patients received a pulmonary function test.

Results: Of the 137 patients who received a pulmonary function test two weeks after discharge, $51.8 \%$ were male, and the mean age was 47 years. Only $19.7 \%$ of the patients were identified as having severe novel coronavirus pneumonia. The pulmonary function test showed that for a small number of patients $((\mathrm{FEV} 1 / \mathrm{FVC}) / \%<70 \%$,) the mean ICV and FVC was $2.4 \pm 0.7 \mathrm{~L}, 3.2 \pm 0.8 \mathrm{~L}$, respectively. In severe cases, $88.9 \%$ of patients had an IVC $<80 \%$ of the predicted value and $55.6 \%$ of patients had an FVC $<80 \%$ of the predicted value. The MEF25, MEF50, and MEF75 $<70 \%$ values were $55.6 \%, 40.7 \%$, and $25.9 \%$, respectively. In the non-severe group, $79.1 \%$ of patients has an IVC $<80 \%$ of the predicted value, and $16.4 \%$ of patients had an FVC $<80 \%$ of the predicted value. The mean MEF25, MEF50, and MEF75 $<70 \%$ values were $57.3 \%$, $30 \%$, and $13.6 \%$, respectively.

Conclusions: In this study, the results suggest that the pulmonary function of patients with 2019 novel coronavirus (COVID-19)-induced pneumonia manifested as restrictive ventilation disorder and small airway obstruction. The incidence was increased among critically ill patients.

Trial registration number: ChiCTR2000029866.

\section{Introduction}

2019 novel coronavirus-induced pneumonia (NCIP) is a viral infectious disease that is now a widespread concern (1-3). The most severe symptom of 2019-nCoV is severe acute respiratory syndrome, and patients die quickly (1). The large-scale injury of type II alveolar epithelial cells induces pulmonary fibrosis by cytokine storm and immunopathology(4). Pulmonary fibrotic changes are occasionally observed in response to viral infections, and pulmonary fibrosis can occasionally be seen as a consequence of several respiratory viral infections, such as Middle East respiratory syndrome (MERS) and avian influenza(5). In particular, in the 2003 epidemic of severe acute respiratory syndrome (SARS), it was noticed that many patients who survived the severe illness developed residual pulmonary fibrosis with increased severity seen in older patients(6). Varying degrees of fibrosis have also been observed in autopsies of fatal COVID-19 cases(7). In the later period of COVID-19-induced illness, the infection mainly manifests as organized pneumonia and fibrosis in clinical findings and radiography(8). The pulmonary function of patients with 2019 novel coronavirus (COVID-19)-induced pneumonia has not yet been 
reported. The purpose of this study is to present pulmonary function and treatment strategies for patients with 2019 novel coronavirus pneumonia (COVID-19)-induced pneumonia.

\section{Methods}

\section{Study design and patients}

This was a retrospective, observational, single-center study. The research was registered in the Chinese Clinical Trial Registry(ChiCTR2000029866). Ethics approval was granted by the Hospital Ethics Committee of Enze Hospital of Taizhou Enze Medical Center (Group). Laboratory-confirmed cases of COVID-19 who improved and were discharged were consecutively included. Written informed consent was obtained from all participants before inclusion.

Diagnostic criteria: Laboratory (nucleic acid)-confirmed infection with COVID-19 and lung involvement confirmed by chest imaging. Discharge criteria: 1). The temperature returned to normal for at least 3 days. 2). Significant improvement in respiratory symptoms. 3). Imaging study showed lung significant inflammatory absorption.4). Two consecutive COVID-19-negative respiratory pathogen nucleic acid detection results (sampling time interval of at least 1 day). Exclusion criteria: 1). Pregnancy. Diagnostic criteria for severe patients: Any of the following: respiratory distress (respiratory rate (RR) $>30$ breaths/min at rest), mean oxygen saturation $\leq 93 \%$; arterial oxygen pressure/oxygen concentration $\left(\mathrm{PaO}_{2} / \mathrm{FiO}_{2}\right) \leq 300 \mathrm{mmHg}(9)$.

\section{Pulmonary Function Test}

The pulmonary ventilation function test method was performed in accordance with the operation code of the American Thoracic Association pulmonary function test via an automatic pulmonary function testing system (Made in Germany)(10). Pulmonary function tests mainly measure the forced expiratory volume in the first second (FEv1), Forced Inspiratory volume (ICV), Forced expiratory volume in the first second as a percentage of the expected value (FEv1/IVC), MEF75 (maximum expiratory volume at $75 \%$ of the vital capacity airflow rate), MEF50 (maximum expiratory flow rate at $50 \%$ of the vital capacity), and MEF25 (maximum expiratory flow rate at $25 \%$ of the vital capacity). Each test was repeated 3 times), and the best figure and data were used. FEV1/FVC $<70 \%$ was defined as obstructive ventilation dysfunction, FVC or IVC $<80 \%$ of the predicted value indicated restrictive ventilation dysfunction, and a value $<70 \%$ of the predicted value defined impaired MEF $25-75 \%$ values.

\section{Data Collection}

All patient data were extracted from electronic medical records, which included age, sex, smoking history, chronic obstructive pulmonary disease history and pulmonary function items. Chest computed tomography was employed for the imaging analysis. 


\section{Statistical Methods}

Data are expressed as the mean \pm standard deviation(SD), median with interquartile range or percent with number of patients. Normality was evaluated by using the Shapiro-Wilk test $(P$ value $>0.05)$.

Comparisons between groups of continuous variables, which were normally distributed, were carried out using Student's t-test or Corrected t-test. Comparisons of other scenarios were performed using the MannWhitney $\mathrm{U}$ test. Comparisons of categorical variables were performed using the $\chi 2$ test or Fisher test. $P$ values $<0.05$ were considered significant. All statistical analyses were performed by SPSS 26.0.

\section{Results}

A total of 137 patients with COVID-19 underwent pulmonary function tests after two weeks of hospital discharge. No patients used ventilator or extracorporeal membrane oxygenation (ECMO). The mean age was 47 years, and $51.8 \%$ were male. Among all patients, 6 patients $(4.4 \%)$ had a smoking habit, and 2 $(1.5 \%)$ patients had a history of chronic obstructive pulmonary disease (Table 1 ). The ICV and FVC values were reduced more significantly in severe cases than mild cases $(P<0.05)$, and the mean ICV and FVC values were $2.4 \pm 0.7$. The FEV1/FVC \% value was $82.7 \pm 7.4 \%$ (Table 1 ). 
Table 1

Baseline characteristics and pulmonary function items of 137 patients with COVID-19

\begin{tabular}{|lllll|}
\hline & All patients & Non-severe & Severe & \\
\hline Characteristics & $(\mathrm{N}=137)$ & $(\mathrm{N}=110)$ & $(\mathrm{N}=27)$ & $\mathrm{P}^{\mathrm{a}}$ \\
\hline Age, Mean \pm SD, year & $47 \pm 13$ & $46 \pm 13$ & $52 \pm 12$ & 0.018 \\
\hline Male sex - no./total no. (\%) & $71 / 137$ & $55 / 110$ & $16 / 27$ & 0.388 \\
\hline Smoking history, no./total no. (\%) & $(51.8 \%)$ & $(50.0 \%)$ & $(59.3 \%)$ & \\
\hline Chronic obstructive pulmonary disease, & $6 / 137$ & $5 / 110$ & $1 / 27$ & 1.000 \\
\hline no./total no. (\%) & $(4.4 \%)$ & $(5.5 \%)$ & $(3.7 \%)$ & \\
\hline Pulmonary Function & $2 / 137$ & $2 / 110$ & 0 & $\mathrm{NA}$ \\
\hline IVC/L & & $(1.8 \%)$ & & \\
\hline FVC/L & $2.4 \pm 0.7$ & $2.5 \pm 0.7$ & $2.1 \pm 0.6$ & 0.003 \\
\hline FEV ${ }_{1} / \mathrm{L}$ & $3.2 \pm 0.8$ & $3.2 \pm 0.7$ & $2.7 \pm 0.8$ & 0.000 \\
\hline (FEV $\left.{ }_{1} / \mathrm{FVC}\right) / \%$ & $2.6 \pm 0.7$ & $2.7 \pm 0.6$ & $2.2 \pm 0.7$ & 0.001 \\
\hline MEF25 & $82.7 \pm 7.4$ & $83.1 \pm 7.3$ & $81.4 \pm 7.9$ & 0.527 \\
\hline MEF50 & $1.2 \pm 0.6$ & $1.2 \pm 0.6$ & $1.1 \pm 0.6$ & 0.266 \\
\hline MEF75 & $3.6 \pm 1.3$ & $3.6 \pm 1.2$ & $3.2 \pm 1.6$ & 0.084 \\
\hline MEF75-85 & $6.0 \pm 1.9$ & $6.1 \pm 1.8$ & $5.3 \pm 2.2$ & 0.061 \\
\hline PEF & $6.1 \pm 2.1$ & $6.3 \pm 2.0$ & $5.4 \pm 2.3$ & 0.065 \\
\hline a $\chi^{2}$ test, Fisher's exact test, Student's t-test and Mann-Whitney U Test & & 0.117 \\
\hline
\end{tabular}

Furthermore, $81.0 \%$ of patients had an IVC $<80 \%$ of the predicted value and $24.1 \%$ of patients had an FVC $<80 \%$ of the predicted value. In severe cases, $88.9 \%$ of patients had an IVC $<80 \%$ of the predicted value and $55.6 \%$ of patients had an FVC $<80 \%$ of the predicted value. The MEF25, MEF50, and MEF75 < $70 \%$ values were $55.6 \%, 40.7 \%$, and $25.9 \%$, respectively. In the non-severe group, $79.1 \%$ of patients had an IVC $<80 \%$ of the predicted value, and $16.4 \%$ of patients had an FVC $<80 \%$ of the predicted value. The mean MEF25, MEF50, and MEF $75<70 \%$ values were $57.3 \%, 30 \%$, and $13.6 \%$, respectively, mainly indicating small airway dysfunction (Fig. 1).

\section{Discussion}


Approximately 200 million people develop viral pneumonia worldwide each year.(11) In the past 10 years, in addition to SARS coronavirus, avian influenza virus (H1N1, H7N9) and Middle East respiratory syndrome coronavirus have periodically had outbreaks(12-14). In patients with viral pneumonia, postARDS pulmonary fibrosis and pulmonary dysfunction are not a rare complication(14). In patients followed up for years, the rate of post-ARDS fibrosis and pulmonary dysfunction of patients with influenza are $10 \%$ and $54.2 \%(14,15)$. In this study, we found reduced ICV and FVC values in $81.0 \%$ of patients. Impaired MEF25-75\% values mainly showed small airway dysfunction (Fig. 1). Our results showed that most patients displayed restrictive ventilatory dysfunction. The ICV decrease was greater than the FVC decrease, indicating that the virus is more harmful to the alveoli than the airways. Preliminary evidence suggests that these lung function abnormalities improve over time (16). Recently, a study described the recovery of lung injury in SARS patients over the past 15 years (17). The results suggest that pulmonary function can be improved to a greater extent when the acute phase of infectious viral pneumonia is effectively managed. In this study, the patients were followed for 2 weeks after hospital discharge. The rate of pulmonary dysfunction disorder was high and consistent with the imaging results(Fig. 2). Of course, the two-week follow-up time is relatively short. Most patients' lung function and pulmonary fibrosis can be restored(17). These patients should continue to be monitored for any changes in condition and symptoms.

Currently, there is no evidence-based foundation for treating pulmonary fibrosis caused by viral pneumonia with medications. Nintedanib is a tyrosine kinase inhibitor that is recommended for idiopathic pulmonary fibrosis (IFP). It exerts anti-pulmonary fibrosis activity by inhibiting fibroblast growth factor receptor (FGFR), platelet-derived growth factor receptor (PDGFR), and vascular endothelial growth factor receptor (VEGFR). Studies in mice found that nintedanib reduces high tidal volume mechanical ventilation-augmented epithelial mesenchymal transition and pulmonary fibrosis after bleomycin-induced acute lung injury(18). Nintedanib has not been used in pulmonary fibrosis patients with viral pneumonia treatment, but more clinical research is needed. Moreover, pulmonary rehabilitation, such as breathing exercises and pulmonary function tests, is necessary for patients with pulmonary fibrosis caused by COVID-19-induced pneumonia.

\section{Conclusions}

In summary, we found that the frequency of pulmonary dysfunction in patients with COVID-19-induced pneumonia is relatively high, manifesting as restrictive dysfunction and small airway injury. We need to continue to pay attention to pulmonary function and strengthen pulmonary function monitoring and rehabilitation treatment if necessary.

\section{List Of Abbreviations}

COVID-19(2019 novel coronavirus), NCIP(2019 novel coronavirus-induced pneumonia), FEv1(Forced expiratory volume in the first second), IVC(Forced Inspiratory volume), FVC(Forced vital capacity), MEF75(Maximum expiratory volume at 75\% vital capacity), MEF50(Maximum expiratory flow rate at 50\% 
vital capacity), MEF25(Maximum expiratory flow rate at 25\% vital capacity), IFP(Idiopathic Pulmonary Fibrosis), PEF(Peak Expiratory Flow)

\section{Declarations}

\section{Ethics approval and consent to participate}

Ethics approval was granted by Hospital Ethics Committee of Enze Hospital of Taizhou Enze Medical Center (Group) (No. K20200204). Written informed consent was obtained from all participators before inclusion.

\section{Consent for publication}

Written informed consent was obtained from all participators before inclusion.

\section{Availability of data and materials}

All data generated during this study are included in this published article [supplementary table1].

\section{Competing interests}

The authors declare that they have no competing interests.

\section{Authors' contributions}

Conception and design: LDQ, JYP; patient treatment and collection of data: CX, MLH,SJ, LZ, WGX,YJS; analysis and interpretation: JYP, LRH; drafting of the manuscript for important intellectual content: JYP; revision of the final manuscript: $L R H, L D Q, W X M$. All authors read and approved the manuscript.

\section{Acknowledgements}

Acknowledgments to all physicians and nurses in Taizhou Public Health Center.

\section{Funding}

The Science and Technology Project of Taizhou(No. 1902ky02 , 1902ky27); The Natural Science Foundation of Zhejiang Province(Y2OH150029).

\section{References}

1. Wang D, Hu B, Hu C, Zhu F, Liu X, Zhang J, et al. Clinical Characteristics of 138 Hospitalized Patients With 2019 Novel Coronavirus-Infected Pneumonia in Wuhan, China. JAMA. 2020.

2. Huang C, Wang Y, Li X, Ren L, Zhao J, Hu Y, et al. Clinical features of patients infected with 2019 novel coronavirus in Wuhan, China. Lancet. 2020. 
3. Roujian Lu XZ, Juan Li, Peihua Niu, Bo Yang, Honglong Wu, Wenling Wang, Hao Song, Baoying Huang, Na Zhu, Yuhai Bi, Xuejun Ma FZ, Liang Wang, Tao Hu, Hong Zhou, Zhenhong Hu, Weimin Zhou, Li Zhao, Jing Chen, Yao Meng, Ji Wang, Yang Lin, Jianying Yuan ZX, Jinmin Ma, William J Liu, Dayan Wang, Wenbo Xu, Edward C Holmes, George F Gao, Guizhen Wuฯ, Weijun Chen,, Weifeng Shi WT. Genomic characterisation and epidemiology of 2019 novel coronavirus: implications for virus origins and receptor binding. Neural Regen Res. 2020.

4. Channappanavar R, Perlman S. Pathogenic human coronavirus infections: causes and consequences of cytokine storm and immunopathology. Semin Immunopathol. 2017;39(5):529-39.

5. Li XY, Sun B, Wang CT, He HY, Zhang CY, Ding Y, et al. A follow-up study on acute respiratory distress syndrome survivors after extracorporeal membrane oxygenation by pulmonary high-resolution $\mathrm{CT}$. Arch Iran Med. 2015;18(1):6-11.

6. Venkataraman T, Frieman MB. The role of epidermal growth factor receptor (EGFR) signaling in SARS coronavirus-induced pulmonary fibrosis. Antiviral Res. 2017;143:142-50.

7. Tian S, Hu W, Niu L, Liu H, Xu H, Xiao SY. Pulmonary pathology of early phase 2019 novel coronavirus (COVID-19) pneumonia in two patients with lung cancer. J Thorac Oncol. 2020.

8. Ye Z, Zhang Y, Wang Y, Huang Z, Song B. Chest CT manifestations of new coronavirus disease 2019 (COVID-19): a pictorial review. Eur Radiol. 2020.

9. China NHCo. The guidelines for the diagnosis and treatment of novel coronavirus (2019-nCoV) infection(Trial Version 5). 2020.

10. Standardization of Spirometry, 1994 Update. American Thoracic Society. Am J Respir Crit Care Med. 1995;152(3):1107-36.

11. Ruuskanen O, Lahti E, Jennings LC, Murdoch DR. Viral pneumonia. Lancet. 2011;377(9773):1264-75.

12. Das KM, Lee EY, Al Jawder SE, Enani MA, Singh R, Skakni L, et al. Acute Middle East Respiratory Syndrome Coronavirus: Temporal Lung Changes Observed on the Chest Radiographs of 55 Patients. AJR Am J Roentgenol. 2015;205(3):W267-74.

13. Chen J, Wu J, Hao S, Yang M, Lu X, Chen X, et al. Long term outcomes in survivors of epidemic Influenza A (H7N9) virus infection. Sci Rep. 2017;7(1):17275.

14. Liu W, Peng L, Liu H, Hua S. Pulmonary Function and Clinical Manifestations of Patients Infected with Mild Influenza A Virus Subtype H1N1: A One-Year Follow-Up. PLoS One. 2015;10(7):e0133698.

15. Mineo G, Ciccarese F, Modolon C, Landini MP, Valentino M, Zompatori M. Post-ARDS pulmonary fibrosis in patients with H1N1 pneumonia: role of follow-up CT. Radiol Med. 2012;117(2):185-200.

16. Chan KS, Zheng JP, Mok YW, Li YM, Liu YN, Chu CM, et al. SARS: prognosis, outcome and sequelae. Respirology. 2003;8 Suppl:S36-40.

17. Zhang P, Li J, Liu H, Han N, Ju J, Kou Y, et al. Long-term bone and lung consequences associated with hospital-acquired severe acute respiratory syndrome: a 15-year follow-up from a prospective cohort study. Bone research. 2020;8:8. 
18. Li LF, Kao KC, Liu YY, Lin CW, Chen NH, Lee CS, et al. Nintedanib reduces ventilation-augmented bleomycin-induced epithelial-mesenchymal transition and lung fibrosis through suppression of the Src pathway. J Cell Mol Med. 2017;21(11):2937-49.

\section{Figures}
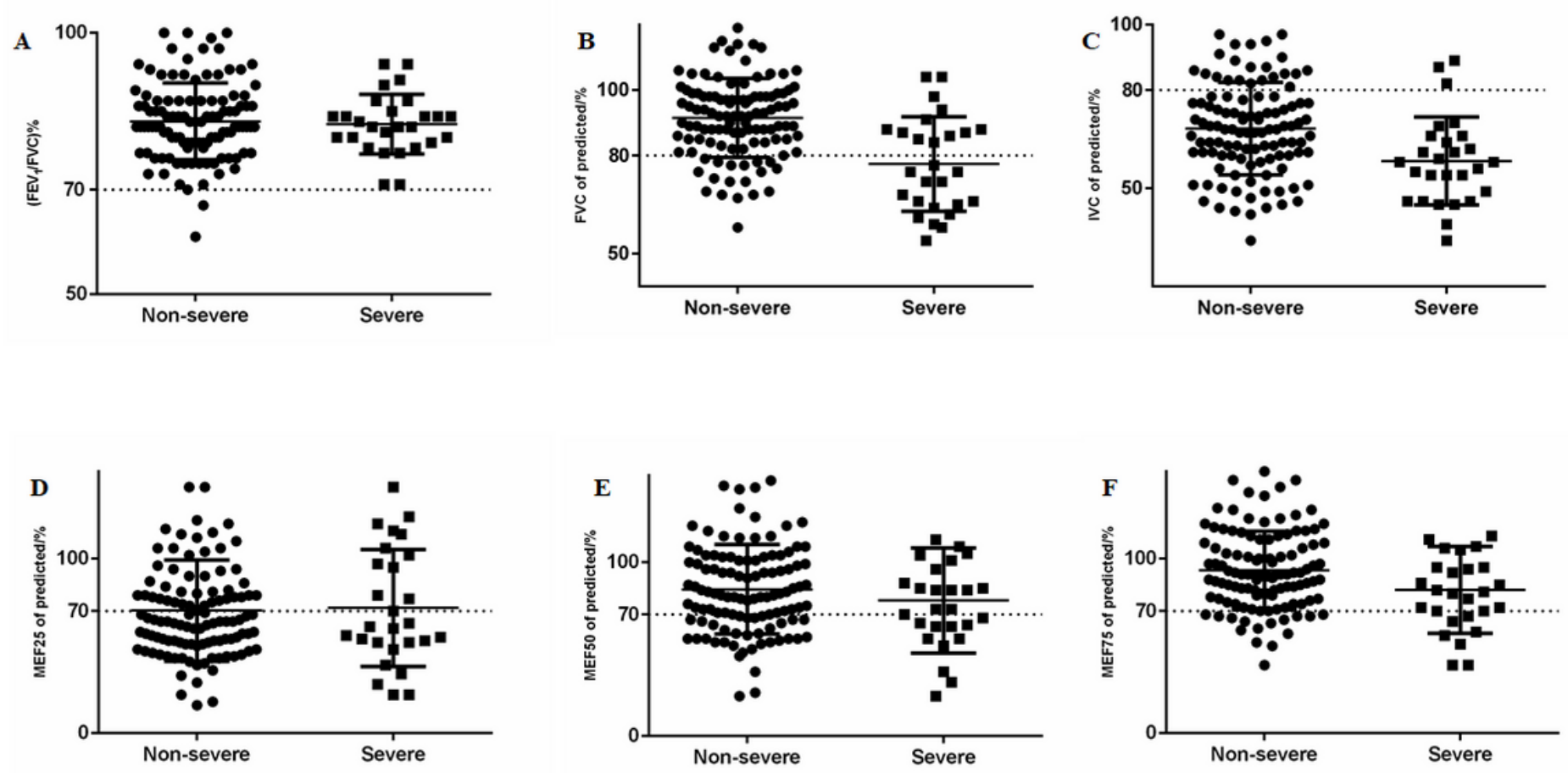

Figure 1

Pulmonary function items are shown. Patient (FEV1/FVC) $\%<70 \%(A) ; 24.1 \%$ of patients had an FVC $<80 \%$ of predicted (B); $81.0 \%$ of patients had an IVC $<80 \%$ of predicted (C); the mean MEF25, MEF50, and MEF75 $<70 \%$ was $56.9 \%, 32.1 \%$, and $16.1 \%$, respectively $(D, E, F)$.

A

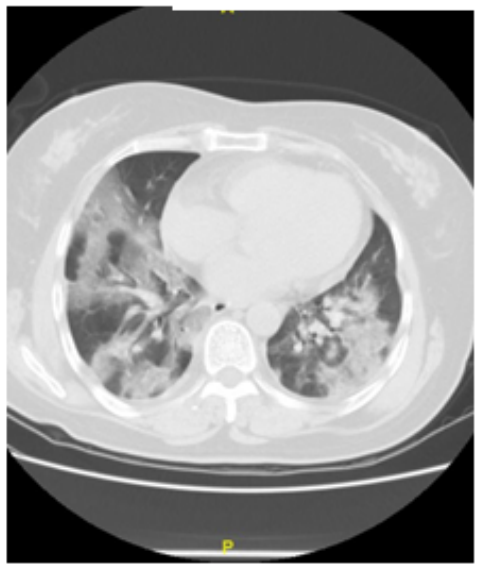

B

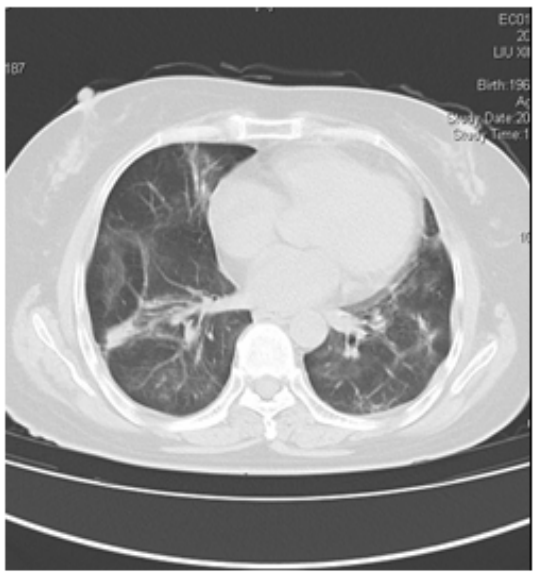

C

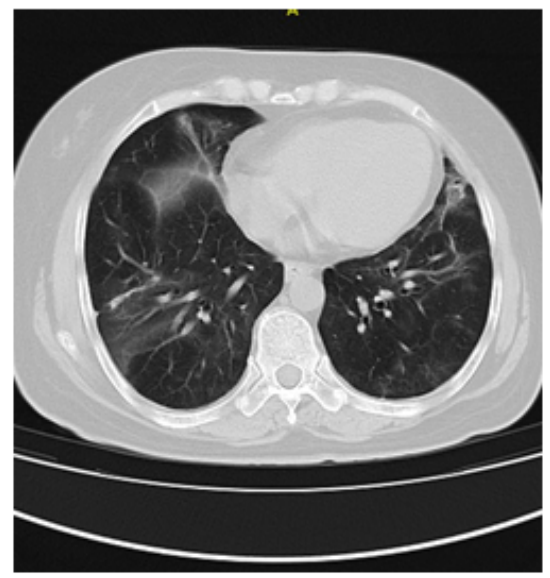


Figure 2

Chest CT of a critically ill patient on admission (A), discharge (B), and 2 weeks after discharge (C). 